\title{
Self-assembly of silk-like protein into nanoscale bicontinuous networks under phase-separation conditions
}

Piotr Batys ${ }^{1,2 *}$, Dmitrii Fedorov ${ }^{2}$, Pezhman Mohammadi ${ }^{3}$,Laura Lemetti ${ }^{2}$, Markus B. Linder ${ }^{2}$, Maria Sammalkorpi ${ }^{2,4}$ *

${ }^{1}$ Jerzy Haber Institute of Catalysis and Surface Chemistry, Polish Academy of Sciences, Niezapominajek 8, PL-30239 Krakow, Poland

${ }^{2}$ Department of Bioproducts and Biosystems, School of Chemical Engineering, Aalto University, FI-00076 Aalto, Finland

${ }^{3}$ VTT Technical Research Centre of Finland Ltd. VTT, FI-02044 Espoo, Finland

${ }^{4}$ Department of Chemistry and Materials Science, School of Chemical Engineering, Aalto University, FI-00076 Aalto, Finland

*Corresponding authors:

Piotr Batys (piotr.batys@ikifp.edu.pl), Maria Sammalkorpi (maria.sammalkorpi@aalto.fi)

\section{The Supplementary Information consist of the following content:}

Final conformations and conformation time evolution for the different initial configurations of CBM-AQ3-CBM in the 200 ns molecular dynamics simulations are presented as Fig. S1 Fig. $\mathrm{S} 2$. presents the corresponding radius of gyration time evolution. Frictional ratio distribution of the CMB-AQ12-CBM proteins as determined from the analytical ultracentrifuge (AUC) data is available as Fig. S3. In Fig. S4, the final $200 \mathrm{~ns}$ molecular dynamics simulation conformations resulting from the different initial configurations of concentrated CBM-AQ3CBM solution $(200 \mathrm{mg} / \mathrm{ml})$ and their contact maps are presented. Table $\mathrm{S} 1$ lists the number of repeat runs corresponding to each simulation system, and Tables S2 and S3 the diffusion coefficients calculated for the individual protein simulations and the multiprotein systems, respectively. 

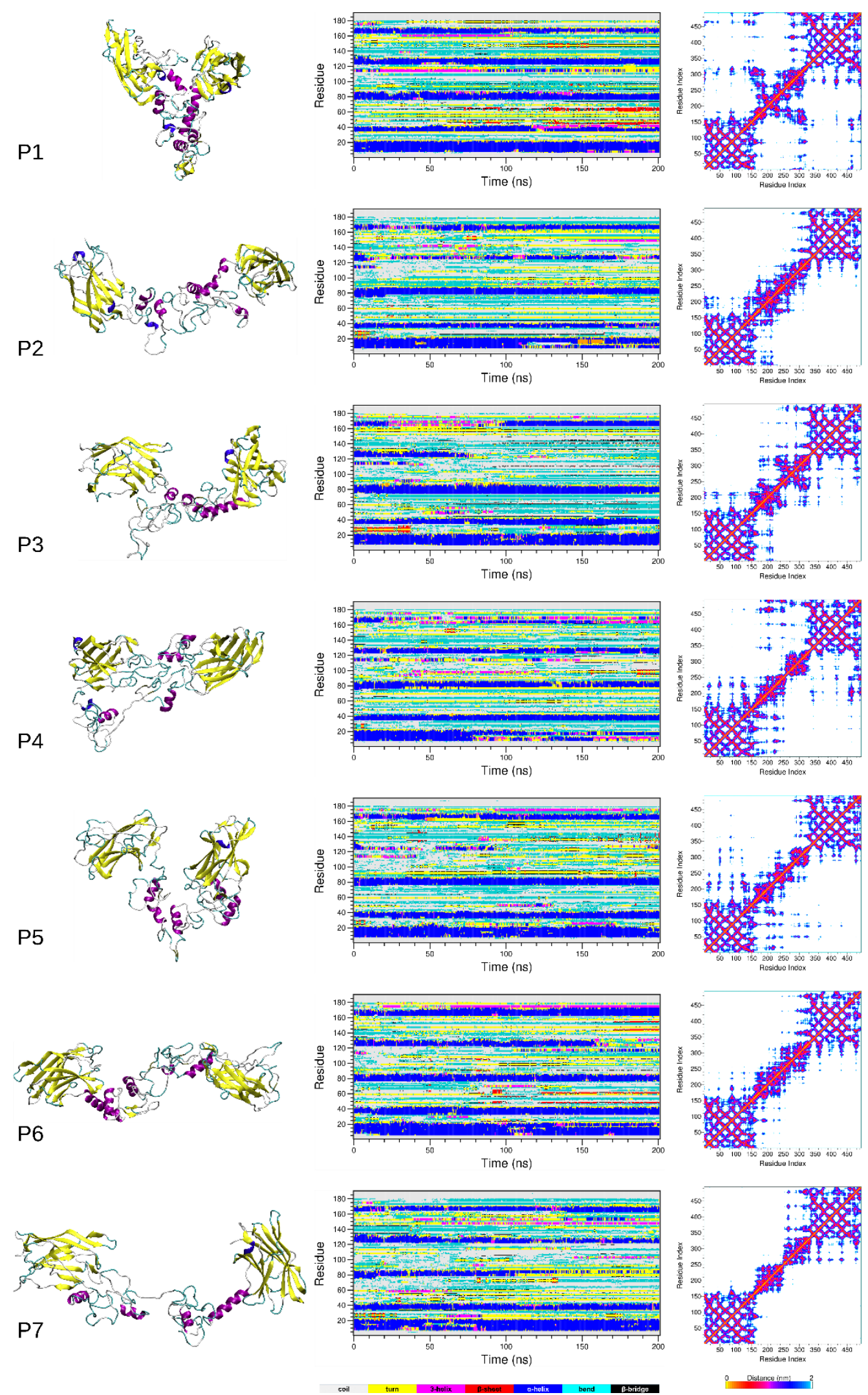

Fig. S1. Final conformations and conformation time evolution for the different initial configurations of CBM-AQ3-CBM in the 200 ns of molecular dynamics simulations. From left to right: molecule identifier (from P1 to P7), final conformation, secondary structure evolution of the repetitive part (without CMBs), and residue contact map. 


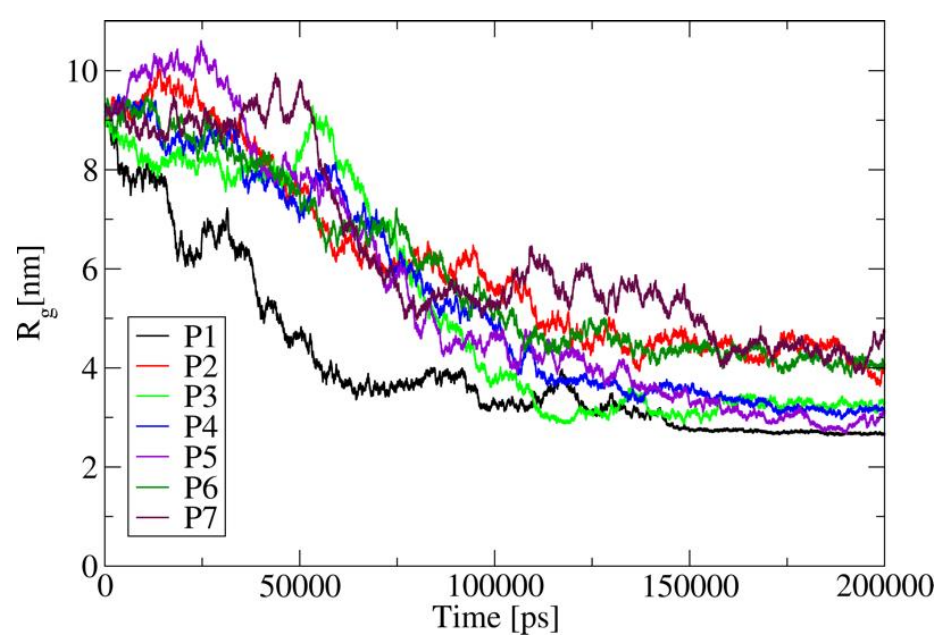

Fig. S2. Radius of gyration changes of different initial configurations of CBM-AQ3-CBM after $200 \mathrm{~ns}$ of molecular dynamics simulations.

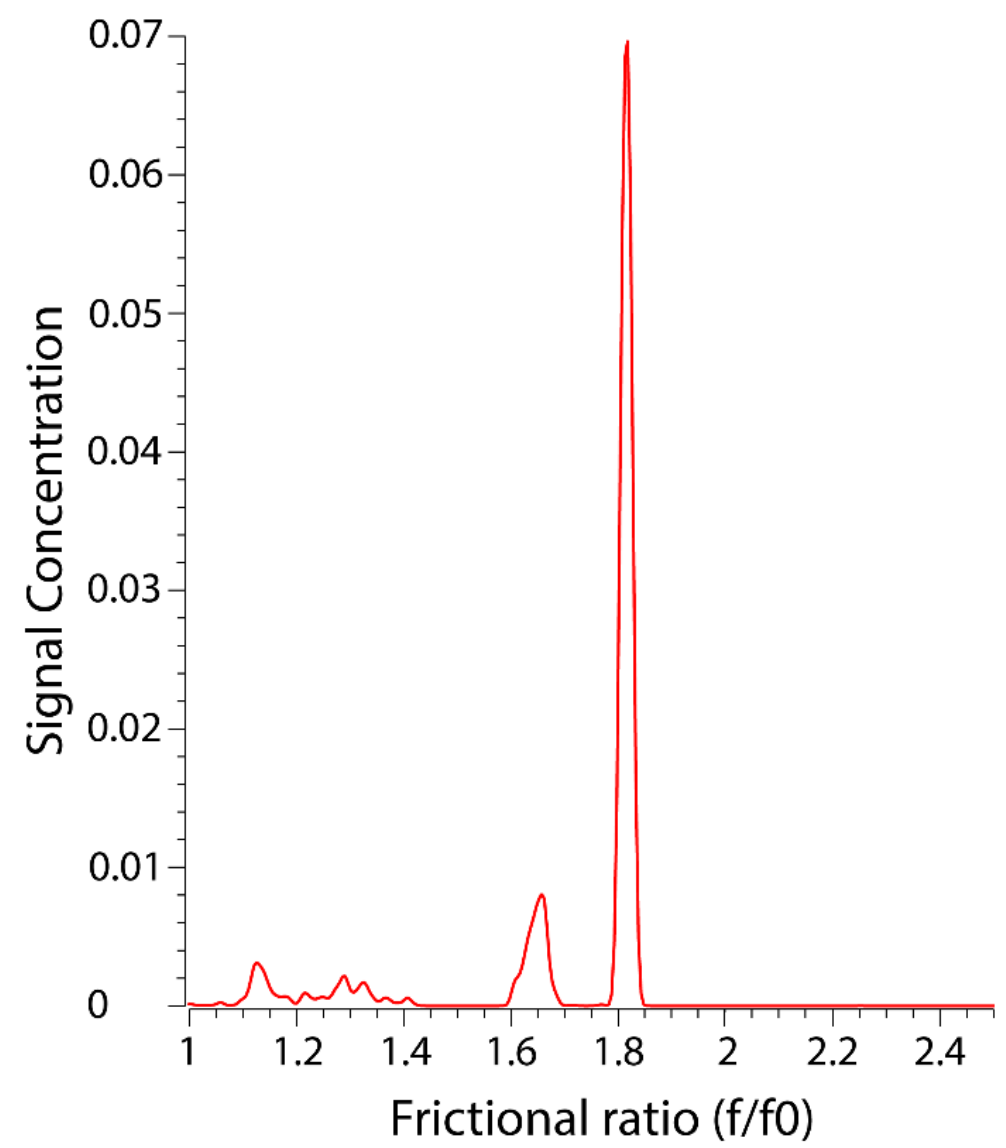

Fig. S3. Frictional ratio distribution determined via AUC experiments for CMB-AQ12-CBM. 

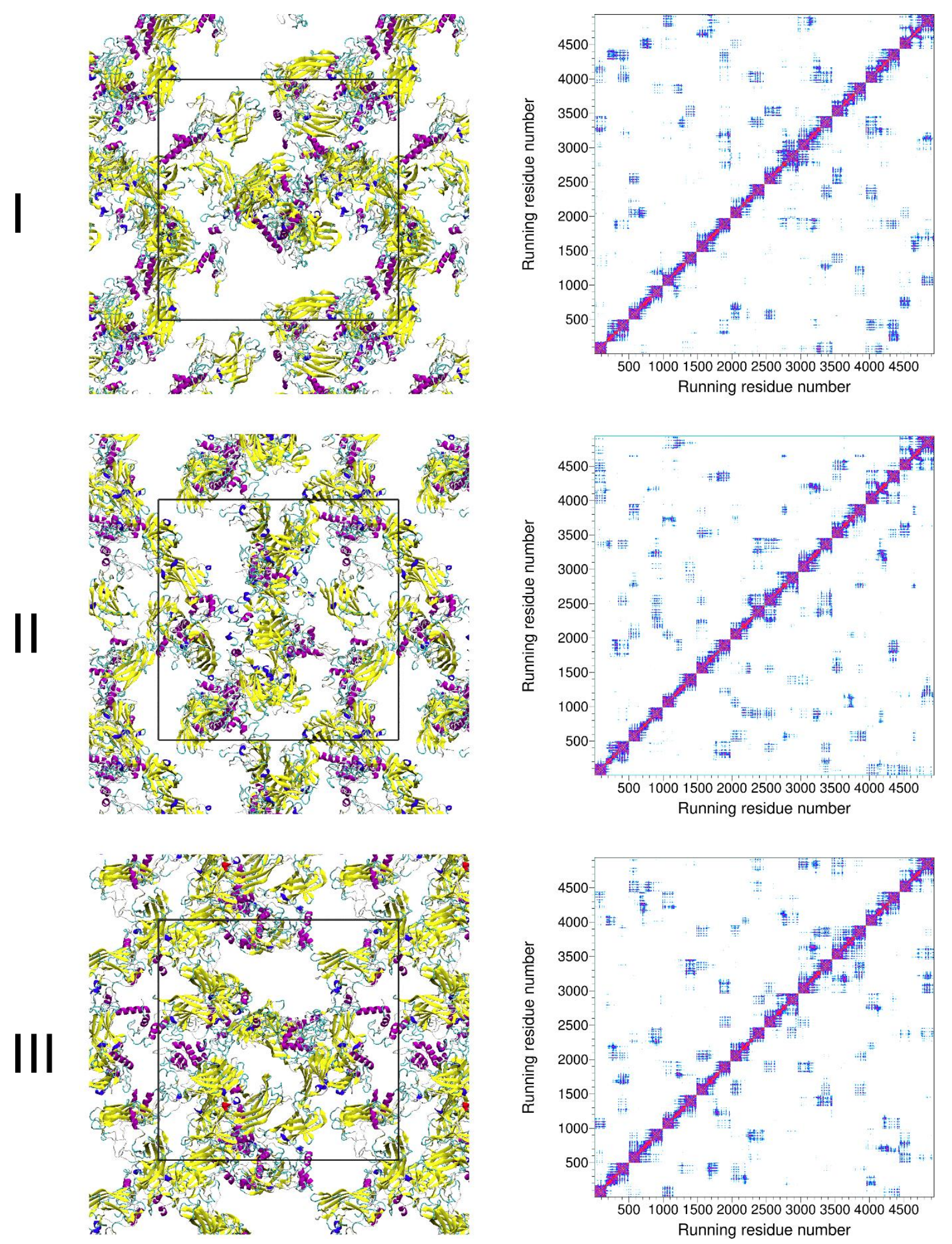

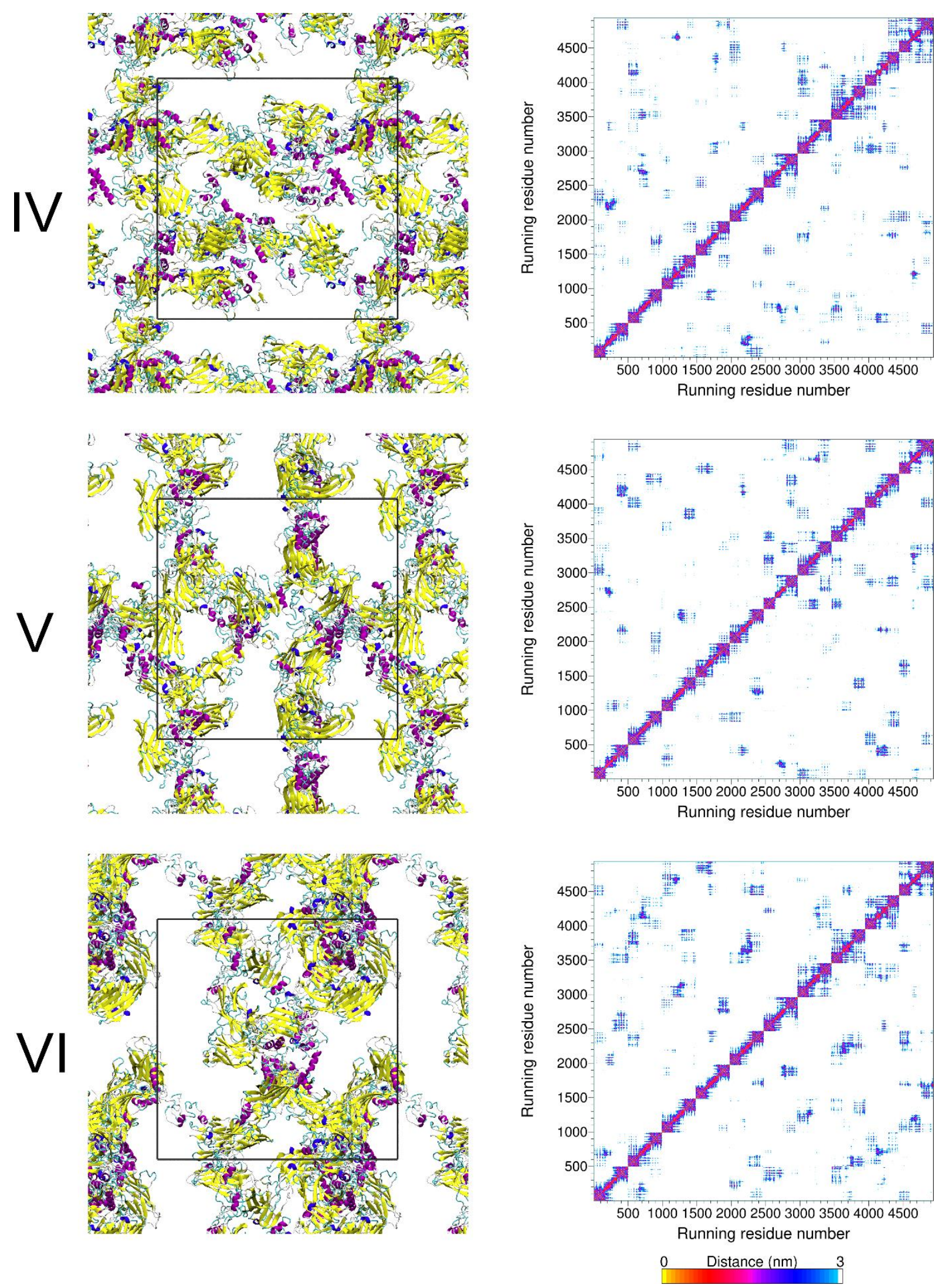

Fig. S4. Final $200 \mathrm{~ns}$ molecular dynamics simulation conformations resulting from the different initial configurations of concentrated CBM-AQ3-CBM solution $(200 \mathrm{mg} / \mathrm{ml})$. From left to right: molecule identifier (from I to VI), final conformation, and the corresponding residue contact map. 
Table S1. Number of molecules with different conformation (from P1 to P7, see Fig. S1) in different initial configurations of highly concentrated solutions (from I - VI, see Fig. S4) used in MD simulations. Total number of proteins in a box equals 10 for each initial configuration and the size of the simulation box was $16.5 \times 16.5 \times 16.5 \mathrm{~nm}^{3}$.

\begin{tabular}{|l|c|c|c|c|c|c|}
\hline & I & II & III & IV & V & VI \\
\hline P1 & 1 & 2 & 2 & 1 & 1 & 1 \\
\hline P2 & 2 & 2 & 2 & 2 & 1 & 1 \\
\hline P3 & 2 & 1 & 1 & 1 & 2 & 1 \\
\hline P4 & 1 & 1 & 1 & 2 & 1 & 1 \\
\hline P5 & 1 & 1 & 2 & 1 & 1 & 2 \\
\hline P6 & 2 & 2 & 1 & 1 & 2 & 2 \\
\hline P7 & 1 & 1 & 2 & 2 & 2 & 2 \\
\hline
\end{tabular}

Table S2. Diffusion coefficients determined from MD simulations of single protein systems (from P1 to P7, see Fig. S1).

\begin{tabular}{|c|c|}
\hline & $\mathbf{D} \times \mathbf{1 0}^{\mathbf{7}}\left[\mathbf{c m}^{2} / \mathbf{s}\right]$ \\
\hline P1 & $4 \pm 6$ \\
\hline P2 & $8 \pm 1$ \\
\hline P3 & $0.3 \pm 0.06$ \\
\hline P4 & $0.7 \pm 0.6$ \\
\hline P5 & $1.3 \pm 0.3$ \\
\hline P6 & $0.6 \pm 0.1$ \\
\hline P7 & $1.1 \pm 0.3$ \\
\hline Average & $\mathbf{2 . 3} \pm \mathbf{1 . 3}$ \\
\hline
\end{tabular}

Table S3. Diffusion coefficients determined from MD simulations of multiple protein systems (from I to VI, see Fig. S4).

\begin{tabular}{|c|c|}
\hline & $\mathbf{D} \times \mathbf{1 0}^{\mathbf{7}}\left[\mathbf{c m}^{2} / \mathbf{s}\right]$ \\
\hline P1 & $0.47 \pm 0.02$ \\
\hline P2 & $0.47 \pm 0.3$ \\
\hline P3 & $0.3 \pm 0.3$ \\
\hline P4 & $0.8 \pm 0.1$ \\
\hline P5 & $0.1 \pm 0.1$ \\
\hline P6 & $0.6 \pm 0.3$ \\
\hline Average & $\mathbf{0 . 4} \pm \mathbf{0 . 3}$ \\
\hline
\end{tabular}

